\title{
218.
}

\section{A THIRD MEMOIR ON THE PROBLEM OF DISTURBED ELLIPTIC MOTION.}

[From the Memoirs of the Royal Astronomical Society, vol. xxxi, pp. 43-56. Read January 10, 1863.]

THE object of the present Memoir is to obtain the differential equations for determining

$$
\begin{aligned}
& r \text {, the radius vector, } \\
& v \text {, the longitude, } \\
& y \text {, the latitude, }
\end{aligned}
$$

of the disturbed body, when the last two coordinates are measured in respect to an arbitrarily varying plane (which however, to fix the ideas, is called the variable ecliptic) and the departure point or origin of longitudes therein. This is very readily effected by means of an expression for the Vis Viva function given in my "Supplementary Memoir on the Problem of Disturbed Elliptic Motion," Mem. Roy. Ast. Soc., t. xxvinr. pp. 217-234 (1859), [215]. Neglecting the squares of the variations of the variable ecliptic, and also the products of the variations by $\sin y$, or $\frac{d y}{d t}$, then (as might be expected) it is found that the equations for $r$ and $v$ are the same as for a fixed ecliptic, and the equation for $y$ is found in a simple form, which is ultimately reduced to coincide with that obtained for the lunar theory by Laplace in the seventh book of the Mécanique Céleste, and which is used by him to show that the effect of the variation of the ecliptic on the latitude of the Moon (as measured from the variable ecliptic) is insensible. And it is shown conversely how the approximate formula of the Memoir may be obtained by a process similar to that made use of in the Mécanique Céleste.

C. III. 
The position in respect to a fixed plane of reference and origin of longitudes therein, of the variable ecliptic and of the departure point or origin of longitudes therein, are determined by

$$
\begin{aligned}
& \theta^{\prime}, \text { the longitude of node, } \\
& \sigma^{\prime}, \text { the departure of node, } \\
& \phi^{\prime} \text {, the inclination, }
\end{aligned}
$$

where, by the definition of a departure point,

$$
d \sigma^{\prime}-\cos \phi^{\prime} d \theta^{\prime}=0,
$$

and then, in respect to the variable ecliptic and departure point or origin of lougitudes therein, the position of the disturbed body is determined by

$r$, the radius vector,

$v$, the longitude,

$y$, the latitude;

and this being so, then (Supplementary Memoir, pp. 220, 227) the expression for the Vis Viva function is,

where

$$
T=\frac{1}{2}\left\{r^{2}+r^{2}\left(Q^{2}+R^{2}\right)\right\},
$$

$$
\begin{aligned}
& Q=-\dot{y}+\left[\cos \left(v-\sigma^{\prime}\right) \sin \phi^{\prime} \cdot \dot{\theta}^{\prime}-\sin \left(v-\sigma^{\prime}\right) \dot{\phi}^{\prime}\right], \\
& R=\cos y \cdot \dot{v}-\sin y\left[\sin \left(v-\sigma^{\prime}\right) \sin \phi^{\prime} \cdot \dot{\theta}^{\prime}+\cos \left(v-\sigma^{\prime}\right) \dot{\phi}^{\prime}\right],
\end{aligned}
$$

the superscript dots being used to denote differentiation with respect to the time. The last-mentioned expressions may for shortness be denoted by

$$
\begin{aligned}
& Q=-\dot{y}+A, \\
& R=\cos y \cdot \dot{v}-B \sin y .
\end{aligned}
$$

The equations of motion are of course,

$$
\begin{aligned}
& \frac{d}{d t} \frac{d T}{d \dot{r}}-\frac{d T}{d r}=\frac{d V}{d r} \\
& \frac{d}{d t} \frac{d T}{d \dot{v}}-\frac{d T}{d v}=\frac{d V}{d v} \\
& \frac{d}{d t} \frac{d T}{d \dot{y}}-\frac{d T}{d y}=\frac{d V}{d y}
\end{aligned}
$$

where $V=\frac{n^{2} a^{3}}{r}+\Omega$, if $\Omega$ is the disturbing function, taken with Lagrange's sign ( $\Omega=-R$, if $R$ is the disturbing function of the Mécanique Céleste). 
218] A THIRD MEMOIR ON THE PROBLEM OF DISTURBED ELLIPTIC MOTION. 507

To reduce these, we have in the first place

$$
\begin{aligned}
& \frac{d T}{d \dot{r}}=\quad \dot{r} \quad, \quad \frac{d T}{d r}=r\left(Q^{2}+R^{2}\right), \\
& \frac{d T}{d \dot{v}}=r^{2} R \cos y, \quad \frac{d T}{d v}=r^{2}\left(Q \frac{d Q}{d v}+R \frac{d R}{d v}\right)=r^{2} \quad(-Q B-R A \sin y), \\
& \frac{d T}{d \dot{y}}=-r^{2} Q \quad, \quad \frac{d T}{d y}=r^{2} R \frac{d R}{d y} \quad=r^{2} R(-\sin y \cdot \dot{v}-B \cos y) .
\end{aligned}
$$

The equations are thus reduced to

$$
\begin{aligned}
& \frac{d^{2} r}{d t^{2}}-r\left(Q^{2}+R^{2}\right)+\frac{n^{2} a^{3}}{r^{2}} \\
& =\frac{d \Omega}{d r}, \\
& \frac{d}{d t}\left(r^{2} \cos y \cdot R\right)+r^{2} \quad(Q B+R A \sin y)=\frac{d \Omega}{d v}, \\
& \frac{d}{d t}\left(-r^{2} Q \quad\right)+r^{2} R\left(\sin y \frac{d v}{d t}+B \cos y\right)=\frac{d \Omega}{d y},
\end{aligned}
$$

and then substituting for $Q, R$ their values, viz.

$$
\begin{aligned}
& Q=-\frac{d y}{d t}+A \\
& R=\cos y \frac{d v}{d t}-B \sin y
\end{aligned}
$$

we find

where

$$
\begin{array}{ll}
\frac{d^{2} r}{d t^{2}}-r\left\{\cos ^{2} y\left(\frac{d v}{d t}\right)^{2}+\left(\frac{d y}{d t}\right)^{2}\right\}+\frac{n^{2} a^{3}}{r^{2}} & =\frac{d \Omega}{d r}+\mathfrak{A} \\
\frac{d}{d t}\left(r^{2} \cos ^{2} y \frac{d v}{d t}\right) & =\frac{d \Omega}{d v}+\mathfrak{B} \\
\frac{d}{d t}\left(r^{2} \frac{d y}{d t}\right)+r^{2} \cos y \sin y\left(\frac{d v}{d t}\right)^{2} & =\frac{d \Omega}{d y}+\mathfrak{\wp}
\end{array}
$$

in which

$$
\begin{aligned}
& \mathfrak{A}=r\left(-2 A \frac{d y}{d t}-2 B \sin y \cos y \frac{d v}{d t}+A^{2}+B^{2} \sin ^{2} y\right), \\
& \mathfrak{B}=\frac{d}{d t}\left(r^{2} B \sin y \cos y\right)+r^{2}\left(B \frac{d y}{d t}-A \sin y \cos y \frac{d v}{d t}-A B \cos ^{2} y\right), \\
& \mathfrak{E}=\frac{d}{d t}\left(r^{2} A\right) \quad+r^{2}\left(-\left(\cos ^{2} y-\sin ^{2} y\right) B \frac{d v}{d t}+B^{2} \sin y \cos y\right),
\end{aligned}
$$

$$
\begin{aligned}
& A=\cos \left(v-\sigma^{\prime}\right) \sin \phi^{\prime} \frac{d \theta^{\prime}}{d t}+\sin \left(v-\sigma^{\prime}\right) \frac{d \phi^{\prime}}{d t} \\
& B=\sin \left(v-\sigma^{\prime}\right) \sin \phi^{\prime} \frac{d \theta^{\prime}}{d t}+\cos \left(v-\sigma^{\prime}\right) \frac{d \phi^{\prime}}{d t}
\end{aligned}
$$

$\theta^{\prime}, \sigma^{\prime}, \phi^{\prime}$ being given functions of $t$ such that $d \sigma^{\prime}-\cos \phi^{\prime} d \theta^{\prime}=0$.

The foregoing equations of motion are rigorously accurate. 
II.

Neglecting the terms which involve $A^{2}, A B, B^{2}$, we have

$$
\begin{aligned}
\mathfrak{A} & =r\left(-2 A \frac{d y}{d t}-2 B \sin y \cos y \frac{d v}{d t}\right), \\
\mathfrak{B} & =\frac{d}{d t}\left(r^{2} B \sin y \cos y\right)+r^{2}\left(B \frac{d y}{d t}-A \sin y \cos y \frac{d v}{d t}\right), \\
\mathfrak{C} & =\frac{d}{d t}\left(r^{2} A\right) \quad-r^{2}\left(\cos ^{2} y-\sin ^{2} y\right) B \frac{d v}{d t}
\end{aligned}
$$

and if we then neglect also the products of $A$ and $B$ by $y$ and $\frac{d y}{d t}$, we have

$$
\begin{aligned}
& \mathfrak{A}=0, \\
& \mathfrak{B}=0, \\
& \mathfrak{C}=\frac{d}{d t}\left(r^{2} A\right)-r^{2} B \frac{d v}{d t},
\end{aligned}
$$

where it may be noticed that, in order to obtain this last value of 6 , the only neglected term is a term containing $B \sin ^{2} y$.

Now, attending to the values of $A$ and $B$, we have

$$
\begin{aligned}
\frac{d A}{d t} & =\frac{d A}{d v} \frac{d v}{d t}+\frac{d^{\prime} A}{d t} \\
& =-B \frac{d v}{d t}+\frac{d^{\prime} A}{d t}
\end{aligned}
$$

where here and in the sequel $\frac{d^{\prime}}{d t}$ denotes differentiation in regard to $t$ in so far only as it enters through the quantities $\sigma^{\prime}, \theta^{\prime}, \phi^{\prime}$, which determine the position of the variable ecliptic.

Hence

$$
\begin{aligned}
\complement & =2 A r \frac{d r}{d t}+r^{2}\left(-B \frac{d v}{d t}+\frac{d^{\prime} A}{d t}\right)-r^{2} B \frac{d v}{d t} \\
& =2 A r \frac{d r}{d t}+r^{2} \frac{d^{\prime} A}{d t}-2 r^{2} B \frac{d v}{d t}
\end{aligned}
$$

and, as above, $\mathfrak{A}=0, \mathfrak{B}=0$.

Let $r, v, y$ be the values obtained on the supposition that $\mathfrak{E}=0$, and

$$
r+\delta r, v+\delta v, y+\delta y,
$$

the accurate values; the first and second equations show that, neglecting the products of $y$ and $\frac{d y}{d t}$ into $\delta y$ and $\frac{d \delta y}{d t}$, we have $\delta r=0, \delta v=0 ;$ so that the values of $r$ and $v$ are not affected by the variation of the ecliptic. And then, substituting in the third equation $y+\delta y$ in the place of $y$, and for

$$
\cos (y+\delta y) \sin (y+\delta y),=\cos y \sin y+\left(\cos ^{2} y-\sin ^{2} y\right) \delta y,
$$


218] A THIRD MEMOIR ON THE PROBLEM OF DISTURBED ELLIY'TIC MOTION. 509 writing $\cos y \sin y+\delta y$, we have

$$
\frac{d}{d t} \cdot r^{2}\left(\frac{d y}{d t}+\frac{d \delta y}{d t}\right)+r^{2}\left(\frac{d v}{d t}\right)^{2}(\cos y \sin y+\delta y)=\frac{d \Omega}{d y}+\delta \frac{d \Omega}{d y}+\widetilde{\sigma}
$$

or, since the terms independent of $\delta y$ and 5 . must destroy each other, this is

$$
\frac{d}{d t} \cdot r^{2} \frac{d \delta y}{d t}+r^{2}\left(\frac{d v}{d t}\right)^{2} \delta y=\delta \frac{d \Omega}{d y}+\delta
$$

or, as this may be written,

$$
r^{2} \frac{d^{2} \delta y}{d t^{2}}+2 r \frac{d r}{d t} \frac{d \delta y}{d t}+r^{2}\left(\frac{d v}{d t}\right)^{2} \delta y=\delta \frac{d \Omega}{d y}+2 A r \frac{d r}{d t}+r^{2} \frac{d^{\prime} A}{d t}-2 r^{2} B \frac{d v}{d t}
$$

that is,

$$
\frac{d^{2} \delta y}{d t^{2}}+\frac{2}{r} \frac{d r}{d t} \frac{d \delta y}{d t}+\left(\frac{d v}{d t}\right)^{2} \delta y=\frac{1}{r^{2}} \delta \frac{d \Omega}{d y}+2 A \frac{1}{r} \frac{d r}{d t}+\frac{d^{\prime} A}{d t}-2 B \frac{d v}{d t}
$$

or, what is the same thing,

$$
\begin{aligned}
& \frac{d^{2} \delta y}{d t^{2}}+n^{2}\left(1+\frac{3}{2} m^{2}\right) \delta y=\frac{1}{r^{2}} \delta \frac{d \Omega}{d y}+\left\{n^{2}+\frac{3}{2} m^{2} n^{2}-\left(\frac{d v}{d t}\right)^{2}\right\} \delta y \\
&-\frac{2}{r} \frac{d r}{d t} \frac{d \delta y}{d t}+2 A \frac{1}{r} \frac{d r}{d t}+2 B\left(n-\frac{d v}{d t}\right) \\
&-2 B n+\frac{d^{\prime} A}{d t},
\end{aligned}
$$

where as usual $m,=\frac{n^{\prime}}{n}$, is the ratio of the mean motion of the Sun to that of the Moon; the term $\frac{3}{2} m^{2} n^{2} \delta y$ having been added on each side of the equation in order to destroy on the right-hand side the corresponding term arising from $\frac{1}{r^{2}} \delta \frac{d \Omega}{d y}$. In fact, to find the approximate expression for $\frac{d \Omega}{d y}$, we have

$$
\Omega=\frac{m^{\prime} r^{2}}{r^{\prime 3}}\left(\frac{3}{2} \cos ^{2} H-\frac{1}{2}\right)
$$

where $H$ is the angular distance of the Sun and Moon; that is, $\cos H=\cos y \cos \left(v-v^{\prime}\right)$; here

$$
\begin{aligned}
\Omega & =\frac{m^{\prime} r^{2}}{r^{\prime 3}}\left\{\frac{3}{2} \cos ^{2} y \cos ^{2}\left(v-v^{\prime}\right)-\frac{1}{2}\right\} \\
\frac{d \Omega}{d y} & =-\frac{m^{\prime} r^{2}}{r^{\prime 3}} \cdot 3 \sin y \cos y \cos ^{2}\left(v-v^{\prime}\right) \\
& =-\frac{m^{\prime} r^{2}}{r^{\prime 3}}\left(\frac{3}{2}+\frac{3}{2} \cos \left(2 v-2 v^{\prime}\right)\right) \sin y \cos y
\end{aligned}
$$


or, neglecting the periodic quantity $\cos \left(2 v-2 v^{\prime}\right)$, and writing $y$ for $\sin y \cos y$; also putting as usual $m^{\prime}=n^{\prime 2} a^{\prime 3}=m^{2} n^{2} a^{\prime 3}$, and $r^{\prime}=a^{\prime}$, we have

and thence

$$
\frac{d \Omega}{d y}=-m^{2} n^{2} r^{2} \cdot \frac{3}{2} y
$$

or

$$
\delta \frac{d \Omega}{d y}=-m^{2} n^{2} r^{2} \cdot \frac{3}{2} \delta y
$$

$$
\frac{1}{r^{2}} \delta \frac{d \Omega}{d y}=-\frac{3}{2} m^{2} n^{2} \delta y
$$

Substituting this value of $\frac{1}{r^{2}} \delta \frac{d \Omega}{d y}$, and putting also $r=a, \frac{d r}{d t}=0, \frac{d v}{d t}=0$, the equation for $\delta y$ becomes

$$
\frac{d^{2} \delta y}{d t^{2}}+n^{2}\left(1+\frac{3}{2} m^{2}\right) \delta y=-2 B n+\frac{d^{\prime} A}{d t} .
$$

\section{III.}

To deduce the formula, seventh book of the Mécanique Céleste, I proceed as follows:

Putting

we have

$$
\frac{d \theta^{\prime}}{d t}=\frac{1}{\cos \phi^{\prime}} \frac{d \sigma^{\prime}}{d t}
$$

$$
\begin{aligned}
& A \cos \phi^{\prime}=\frac{d \sigma^{\prime}}{d t} \sin \phi^{\prime} \cos \left(v-\sigma^{\prime}\right)-\frac{d \phi^{\prime}}{d t} \cos \phi^{\prime} \sin \left(v-\sigma^{\prime}\right), \\
& B \cos \phi^{\prime}=\frac{d \sigma^{\prime}}{d t} \sin \phi^{\prime} \sin \left(v-\sigma^{\prime}\right)+\frac{d \phi^{\prime}}{d t} \cos \phi^{\prime} \cos \left(v-\sigma^{\prime}\right)
\end{aligned}
$$

which may be written

$$
\begin{aligned}
& A \cos \phi^{\prime}=-\sin v \frac{d}{d t}\left(\sin \phi^{\prime} \cos \sigma^{\prime}\right)+\cos v \frac{d}{d t}\left(\sin \phi^{\prime} \sin \sigma^{\prime}\right) \\
& B \cos \phi^{\prime}=\cos v \frac{d}{d t}\left(\sin \phi^{\prime} \cos \sigma^{\prime}\right)+\sin v \frac{d}{d t}\left(\sin \phi^{\prime} \sin \sigma^{\prime}\right)
\end{aligned}
$$

Laplace in effect assumes that the variations of the ecliptic are given in the form

$$
\begin{aligned}
& \sin \phi^{\prime} \sin \sigma^{\prime}=-\Sigma k \sin (i n t+\epsilon), \\
& \sin \phi^{\prime} \cos \sigma^{\prime}=\Sigma k \cos (i n t+\epsilon),
\end{aligned}
$$

( $i t+\epsilon$ is there written for the argument, $n$ being assumed $=1$ ) where $i, k, \epsilon$ are absolute constants, the quantities $i$ being all very small in comparison with $m^{2}$. Substituting these values, and putting $\cos \phi^{\prime}$ equal to unity, we have

$$
\begin{aligned}
& A=-\Sigma i k \cos (v+i n t+\epsilon) \\
& B=-\Sigma i k \sin (v+i n t+\epsilon)
\end{aligned}
$$


and thence also

$$
\frac{d^{\prime} A}{d t}=n \Sigma i^{2} k \sin (v+i n t+\epsilon)
$$

and

$$
2 n B-\frac{d^{\prime} A}{d t}=-n \Sigma\left(2 i+i^{2}\right) k \sin (v+i n t+\epsilon),
$$

so that the equation for $\delta y$ becomes

$$
\frac{d^{2} \delta y}{d t^{2}}+n^{2}\left(1+\frac{3}{2} m^{2}\right) \delta y+n \Sigma\left(2 i+i^{2}\right) k \sin (v+i n t+\epsilon)=0
$$

or, taking as the independent variable $v(=n t)$ in the place of $t$, this is

$$
\frac{d^{2} \delta y}{d v^{2}}+\left(1+\frac{3}{2} m^{2}\right) \delta y+\frac{1}{n} \Sigma\left(2 i+i^{2}\right) k \sin (v+i v+\epsilon)=0
$$

which is, in fact, Laplace's equation, $n$ being retained instead of being put equal to unity, and $\delta y$ being the part which depends on the variation of the ecliptic, of his $s$.

\section{IV.}

Conversely the equation

$$
\frac{d^{2} \delta y}{d t^{2}}+n^{2}\left(1+\frac{3}{2} m^{2}\right) \delta y=-2 B n+\frac{d^{\prime} A}{d t},
$$

may be obtained by a process similar to Laplace's. Assuming that the Moon and Sun are each of them referred to a fixed plane of reference and origin of longitudes therein, by the coordinates

$$
\begin{aligned}
& u \text {, the reciprocal of the reduced radius vector, } \\
& v \text {, the longitude, } \\
& s \text {, the tangent of the latitude, }
\end{aligned}
$$

for the Moon, and by the corresponding coordinates $u^{\prime}, v^{\prime}, s^{\prime}$ for the Sun, then we have

$$
\frac{d^{2} s}{d v^{2}}+s+\frac{\frac{d s}{d v} \frac{d \Omega}{d v}-s u \frac{d \Omega}{d u}-\left(1+s^{2}\right) \frac{d \Omega}{d s}}{n^{2} u^{2}\left(1+\frac{2}{n^{2}} \int \frac{d \Omega}{u^{2} d v} d v\right)}=0 .
$$

Here, as before,

$$
\Omega=\frac{m^{\prime} r^{2}}{r^{\prime 3}}\left(\frac{3}{2} \cos ^{2} H-\frac{1}{2}\right)
$$

or, as it is now to be written,

$$
\Omega=\frac{m^{\prime}\left(1+s^{2}\right) u^{\prime 3}}{\left(1+s^{\prime 2}\right)^{\frac{3}{2}} u^{2}}\left\{\frac{3}{2}\left(\frac{\cos \left(v-v^{\prime}\right)+s s^{\prime}}{\sqrt{1+s^{2}} \sqrt{1+s^{\prime 2}}}\right)^{2}-\frac{1}{2}\right\}
$$


or, since the second term

$$
\frac{m^{\prime} u^{\prime 3}}{\left(1+s^{\prime 2}\right)^{\frac{3}{2}}}-\frac{1}{2} \frac{1+s^{2}}{u^{2}}
$$

gives, as is immediately seen, no term in

$$
\frac{d s}{d v} \frac{d \Omega}{d v}-s u \frac{d \Omega}{d u}-\left(1+s^{2}\right) \frac{d \Omega}{d s},
$$

we may, in calculating this quantity, write

$$
\begin{aligned}
\Omega & =\frac{m^{\prime}\left(1+s^{2}\right) u^{\prime 3}}{\left(1+s^{\prime 2}\right)^{\frac{3}{2}} u^{2}} \cdot \frac{3}{2}\left(\frac{\cos \left(v-v^{\prime}\right)+s s^{\prime}}{\sqrt{1+s^{2}} \sqrt{1+s^{\prime 2}}}\right)^{2} \\
& =\frac{3}{2} \frac{m^{\prime} u^{\prime 3}}{\left(1+s^{\prime 2}\right)^{\frac{5}{2}} u^{2}}\left(\cos \left(v-v^{\prime}\right)+s s^{\prime}\right)^{2},
\end{aligned}
$$

or, neglecting $s^{\prime 2}$,

$$
\Omega=\frac{3}{2} m^{\prime} u^{\prime 3}\left(\frac{\cos \left(v-v^{\prime}\right)+s s^{\prime}}{u}\right)^{2} .
$$

Hence, putting for a moment

$$
\Theta=\frac{\cos \left(v-v^{\prime}\right)+s s^{\prime}}{u},
$$

we have

$$
\frac{d s}{d v} \frac{d \Omega}{d v}-s u \frac{d \Omega}{d u}-\left(1+s^{2}\right) \frac{d \Omega}{d s}=3 m^{\prime} u^{\prime 3} \cdot \Theta\left\{\frac{d s}{d v} \frac{d \Theta}{d v}-s u \frac{d \Theta}{d u}-\left(1+s^{2}\right) \frac{d \Theta}{d s}\right\}
$$

where the factor in \{\} is

$$
\begin{aligned}
& =\frac{1}{u}\left\{-\frac{d s}{d v} \sin \left(v-v^{\prime}\right)+s\left(\cos \left(v-v^{\prime}\right)+s s^{\prime}\right)-\left(1+s^{2}\right) s^{\prime}\right\} \\
& =\frac{1}{u}\left\{s \cos \left(v-v^{\prime}\right)-\frac{d s}{d v} \sin \left(v-v^{\prime}\right)-s^{\prime}\right\},
\end{aligned}
$$

and the above-mentioned quantity is

$$
=\frac{3 m^{\prime} u^{\prime}}{u^{2}}\left\{\cos \left(v-v^{\prime}\right)+s s^{\prime}\right\}\left\{s \cos \left(v-v^{\prime}\right)-\frac{d s}{d v} \sin \left(v-v^{\prime}\right)-s^{\prime}\right\} ;
$$

or, what is the same thing,

$$
=\frac{3 m^{\prime} u^{\prime 3}}{u^{2}} \cos \left(v-v^{\prime}\right)\left\{s \cos \left(v-v^{\prime}\right)-\frac{d s}{d v} \sin \left(v-v^{\prime}\right)-s^{\prime}\right\} .
$$

Considering now the Sun as moving in the variable ecliptic, its latitude is $=\sin \phi^{\prime} \sin \left(v^{\prime}-\sigma^{\prime}\right)$; that is, we have

$$
s^{\prime}=\sin \phi^{\prime} \sin \left(v^{\prime}-\sigma^{\prime}\right)
$$


and if the Moon were in the variable ecliptic, its latitude would be $\sin \phi^{\prime} \sin \left(v-\sigma^{\prime}\right)$; that is, the latitude, measured from the variable ecliptic, is $=s-\sin \phi^{\prime} \sin \left(v-\sigma^{\prime}\right)$; or, putting

we have

$$
s, \text { the Moon's latitude, measured from the variable ecliptic, }
$$

$$
s=\sin \phi^{\prime} \sin \left(v-\sigma^{\prime}\right)+s,
$$

Hence, disregarding the variations of $\phi^{\prime}, \sigma^{\prime}$, we have

$$
\frac{d s}{d v}=\sin \phi^{\prime} \cos \left(v-\sigma^{\prime}\right)+\frac{d s}{d v}
$$

and substituting these values of $s, \frac{d s}{d v}$, and $s^{\prime}$, we find

$$
\begin{aligned}
& s \cos \left(v-v^{\prime}\right)-\frac{d s}{d v} \sin \left(v-v^{\prime}\right)-s^{\prime} \\
&= s_{1} \cos \left(v-v^{\prime}\right)-\frac{d s_{1}}{d v} \sin \left(v-v^{\prime}\right) \\
&+\sin \phi^{\prime}\left\{\sin \left(v-\sigma^{\prime}\right) \cos \left(v-v^{\prime}\right)-\cos \left(v-\sigma^{\prime}\right) \sin \left(v-v^{\prime}\right)-\sin \left(v^{\prime}-\sigma^{\prime}\right)\right\} \\
&= s, \cos \left(v-v^{\prime}\right)-\frac{d s}{d v} \sin \left(v-v^{\prime}\right)
\end{aligned}
$$

or

$$
\begin{aligned}
\frac{d s}{d v} \frac{d \Omega}{d v} & -s u \frac{d \Omega}{d u}\left(1+s^{2}\right) \frac{d \Omega}{d s} \\
& =\frac{3 m^{\prime} u^{\prime 3}}{u^{2}} \cos \left(v-v^{\prime}\right)\left\{s, \cos \left(v-v^{\prime}\right)-\frac{d s}{d v} \sin \left(v-v^{\prime}\right)\right\}
\end{aligned}
$$

which, neglecting the periodic terms, is

$$
=\frac{3}{2} \frac{m^{\prime} u^{\prime}}{u^{2}} s,
$$

and then

$$
\frac{\frac{d s}{d v} \frac{d \Omega}{d v}-s u \frac{d \Omega}{d u}-\left(1+s^{2}\right) \frac{d \Omega}{d s}}{h^{2} u^{2}\left(1+\frac{2}{h^{2}} \int \frac{d \Omega}{u^{2} d v} d v\right)}=\frac{1}{h^{2} u^{2}} \cdot \frac{3}{2} \frac{m^{\prime} u^{\prime 3}}{u^{2}} s_{1}=\frac{3}{2} \frac{m^{\prime} u^{\prime 3}}{h^{2} u^{4}} s_{;}
$$

which, putting $m^{\prime}=n^{\prime 2} a^{\prime 3}=m^{2} n^{2} a^{\prime 3}, u^{\prime}=\frac{1}{a^{\prime}}, h^{2}=n^{2} a^{4}, u=\frac{1}{a}$, becomes

$$
=\frac{3}{2} m^{2} s \text {; }
$$

so that the differential equation is reduced to

$$
\frac{d^{2} s}{d v^{2}}+s+\frac{3}{2} m^{2} s,=0 .
$$

C. III. 
514 A THIRD MEMOIR ON THE PROBLEM OF DISTURBED ELLIPTIC MOTION. [218

But

$$
\begin{aligned}
& s=\sin \phi^{\prime} \sin \left(v-\sigma^{\prime}\right)+s_{,} \\
& \frac{d s}{d v}=\sin \phi^{\prime} \cos \left(v-\sigma^{\prime}\right)+\frac{d^{\prime}}{d v} \sin \phi^{\prime} \sin \left(v-\sigma^{\prime}\right)+\frac{d s}{d v}, \\
& \frac{d^{2} s}{d v^{2}}=-\sin \phi^{\prime} \sin \left(v-\sigma^{\prime}\right)+\frac{d^{\prime}}{d v} \sin \phi^{\prime} \cos \left(v-\sigma^{\prime}\right)+\frac{d}{d v} \frac{d^{\prime}}{d v} \sin \phi^{\prime} \sin \left(v-\sigma^{\prime}\right)+\frac{d^{2} s}{d v^{2}},
\end{aligned}
$$

where $\frac{d^{\prime}}{d v}$ denotes differentiation in respect to $v$, in so far only as it enters through $\phi^{\prime}$ and $\sigma^{\prime}$ (these are functions of $t$, which is $\left.=\frac{1}{n} v\right)$. Hence

$$
\frac{d^{2} s}{d v^{2}}+s=\frac{d^{2} s}{d v^{2}}+s,+\frac{d^{\prime}}{d v} \sin \phi^{\prime} \cos \left(v-\sigma^{\prime}\right)+\frac{d}{d v} \frac{d^{\prime}}{d v} \sin \phi^{\prime} \sin \left(v-\sigma^{\prime}\right)
$$

but

$$
\begin{aligned}
\frac{d^{\prime}}{d v} \sin \phi^{\prime} \sin \left(v-\sigma^{\prime}\right) & =\frac{1}{n}\left(\sin v \frac{d}{d t} \sin \phi^{\prime} \cos \sigma^{\prime}-\cos v \frac{d}{d t} \sin \phi^{\prime} \sin \sigma^{\prime}\right) \\
& =-\frac{1}{n} A \cos \phi^{\prime} \\
& =-\frac{1}{n} A
\end{aligned}
$$

and thence

$$
\frac{d}{d v} \frac{d^{\prime}}{d v} \sin \phi^{\prime} \sin \left(v-\sigma^{\prime}\right)=-\frac{1}{n} \frac{d A}{d v}-\frac{1}{n} \frac{d^{\prime} A}{d v}=\frac{1}{n} B-\frac{1}{n^{2}} \frac{d^{\prime} A}{d t}
$$

and similarly

$$
\begin{aligned}
\frac{d^{\prime}}{d v} \sin \phi^{\prime} \cos \left(v-\sigma^{\prime}\right) & =\frac{1}{n}\left\{\cos v \frac{d}{d t}\left(\sin \phi^{\prime} \cos \sigma^{\prime}\right)+\sin v \frac{d}{d t} \sin \phi^{\prime} \sin \sigma^{\prime}\right\} \\
& =\frac{1}{n} B \cos \phi^{\prime} \\
& =\frac{1}{n} B
\end{aligned}
$$

Hence

$$
\frac{d^{2} s}{d v^{2}}+s=\frac{d^{2} s}{d v^{2}}+s,+\frac{2}{n} B-\frac{1}{n^{2}} \frac{d^{\prime} A}{d t}
$$

or, putting $v=n t$,

$$
\frac{d^{2} s}{d v^{2}}+s=\frac{1}{n^{2}} \frac{d^{2} s}{d t^{2}}+s,+\frac{2}{n} B-\frac{1}{n^{2}} \frac{d^{\prime} A}{d t}
$$


218] A THIRD MEMOIR ON THE PROBLEM OF DISTURBED ELLIPTIC MOTION. 515 whence, substituting in the equation

$$
\frac{d^{2} s}{d v^{2}}+s+\frac{3}{2} m^{2} s,=0
$$

we have

$$
\frac{d^{2} s}{d t^{2}}+n^{2}\left(1+\frac{3}{2} m^{2}\right) s,+2 n B-\frac{d^{\prime} A}{d t}=0
$$

or putting $s,=y+\delta y$, then

$$
\frac{d^{2} y}{d t^{2}}+n^{2}\left(1+\frac{3}{2} m^{2}\right) y=0
$$

which gives, in the approximation which is being considered, the principal term of the latitude, and then

$$
\frac{d^{2} \delta y}{d t^{2}}+n^{2}\left(1+\frac{3}{2} m^{2}\right) \delta y+2 n B-\frac{d^{\prime} A}{d t}=0
$$

which is the approximate equation previously obtained by the method of the present memoir. 